\title{
Sequence analysis of heparan sulphate and heparin oligosaccharides
}

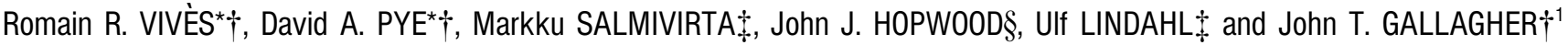 \\ ${ }^{*}$ CRC section of Drug Development and Imaging, Department of Medical Oncology, Paterson Institute for Cancer Research, Christie Hospital, Manchester M20 4BX, U.K., \\ $\uparrow C R C$ and University of Manchester, Department of Medical Oncology, Paterson Institute for Cancer Research, Christie Hospital, Manchester M20 4BX, U.K., \\ \$Department of Medical Biochemistry and Microbiology, Uppsala University, Biomedical Center P.0. Box 575, S-75123 Uppsala, Sweden, and $\lfloor$ Lysosomal Diseases \\ Research Unit, Department of Chemical Pathology, Women's and Children's Hospital, North Adelaide, SA 5006, Australia
}

\begin{abstract}
The biological activity of heparan sulphate (HS) and heparin largely depends on internal oligosaccharide sequences that provide specific binding sites for an extensive range of proteins. Identification of such structures is crucial for the complete understanding of glycosaminoglycan (GAG)-protein interactions. We describe here a simple method of sequence analysis relying on the specific tagging of the sugar reducing end by ${ }^{3} \mathrm{H}$ radiolabelling, the combination of chemical scission and specific enzymic digestion to generate intermediate fragments, and the analysis of the generated products by strong-anion-exchange HPLC. We present full sequence data on microgram quantities
\end{abstract}

of four unknown oligosaccharides (three HS-derived hexasaccharides and one heparin-derived octasaccharide) which illustrate the utility and relative simplicity of the technique. The results clearly show that it is also possible to read sequences of inhomogeneous preparations. Application of this technique to biologically active oligosaccharides should accelerate progress in the understanding of HS and heparin structure-function relationships and provide new insights into the primary structure of these polysaccharides.

Key words: glycosaminoglycan, lysosomal enzymes, sequence.

\section{INTRODUCTION}

Glycosaminoglycans (GAGs) are linear polysaccharides found on most animal cell surfaces as well as in basement membranes and other extracellular matrices. Heparan sulphate (HS) and heparin are the most widely studied members of this family, since they are implicated in many biological functions [1]. However, detailed study of these molecules is made difficult by their highly complex and heterogeneous structure [2,3].

Biosynthesis of heparin and HS involves the formation of a common precursor polysaccharide comprised of alternating $\mathrm{N}$ acetylglucosamine and glucuronic acid units, which subsequently undergoes a stepwise series of modifications [4]. These start with the N-deacetylation/N-sulphation of glucosamine residues, followed by $\mathrm{C}-5$ epimerization of the adjacent glucuronate into iduronate (IdoA). Finally, O-sulphation occurs at C-2 of IdoA and C-6 of glucosamine, with infrequent modifications taking place at C-2 of glucuronic acids and C-3 of $N$-sulphoglucosamine (GlcNS).

Little is known about the mechanism of biosynthetic regulation. However, it is clear that this process is non-random. The stepwise nature of the modification reactions, the substrate specificity of the enzymes involved and the extent of glucosamine $\mathrm{N}$-sulphation lead to a hypervariable, but precise, molecular organization $[2,4]$. In HS, N-sulphation of glucosamine is an incomplete process which only occurs in defined regions of the polysaccharide. This results in the formation of clusters of $\mathrm{N}$ sulphated disaccharides, called S-domains, which then constitute major sites for further modifications. In the mature HS chains, highly sulphated regions alternate with relatively unmodified regions of glucuronic acid- $N$-acetylglucosamine (GlcA-GlcNAc) repeats. In heparin, $\mathrm{N}$-sulphation is much more extensive, and the polysaccharide is therefore enriched in IdoA residues and $O$-sulphate groups, confering to the whole polysaccharide an $\mathrm{S}$ domain-type organization.

Heparin and HS have been implicated in numerous biological activities [1] which are mainly triggered by interactions with a wide range of proteins, such as cofactors of the coagulation cascade, extracellular-matrix components and a variety of cytokines and growth factors $[3,5,6]$. Heparin, because of its ability to bind and enhance the activity of antithrombin, has been used clinically for over half a century as an anticoagulant and antithrombotic agent. However, under physiological conditions, HS is believed to be the actual mediator of ligand binding and activation and thus seems to be the polysaccharide most relevant for study of biological mechanisms.

The protein-binding properties of $\mathrm{HS} /$ heparin are mediated by regions of specific sugar sequence and sulphation pattern in the polymer chains. The most notable example to date is the antithrombin-binding pentasaccharide sequence featuring a unique 3-O-sulphated glucosamine [6]. More recently, studies on fibroblast growth factor $2(\mathrm{FGF}-2)$ have indicated a requirement for iduronate 2-O-sulphate (IdoA,2S) and GlcNS residues for recognition of the growth factor [7,8], but its activation by HS also needs the presence of $6-O$-sulphate groups $[9,10]$. The binding and activation of other growth factors, such as FGF-1, FGF-4 [10,11], hepatocyte growth factor [12] or vascular epithelial cell growth factor [13], were also shown to be dependent on distinct structural features, supporting the existence of ligandspecific sites within HS chains. However, the lack of a convenient method for precise GAG structure analysis has meant that little is known about the exact sequences exerting activity for most ligands of HS and heparin. Classical investigation techniques rely on the utilization of specific enzymic and chemical scission

Abbreviations used: 6Sase, glucosamine 6-O-sulphatase (EC 3.1.6.14); FGF, fibroblast growth factor; GlcA, glucuronic acid; GlcNAc, $N$ acetylglucosamine; GIcNS, $N$-sulphoglucosamine; GAG, glycosaminoglycan; $\Delta$ HexA: $\Delta 4,5$-unsaturated uronic acid; HS, heparan sulphate; I2Sase,

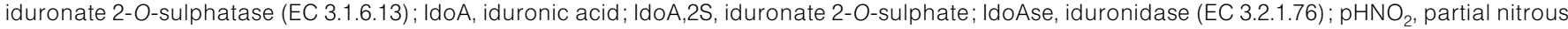
acid; SAX, strong anion exchange.

To whom correspondence should be sent (e-mail jgallagher@picr.man.ac.uk). 
methods and the subsequent analysis of the degradation products. This provides data on the overall composition and domain organization of HS [14-16], but no sequence information. Other analysis methods, such as NMR or MS [17,18] have been used for the structural characterization of short oligosaccharides, but require expertise and equipment incompatible with routine use in most laboratories. HS-degrading lysosomal enzymes have been used to provide information on the structure of the non-reducing end of HS and heparin fragments [19]. In the present study we have used a combined chemical and enzymic-scission protocol for the complete sequencing of oligosaccharides derived from HS and heparin. This method involves specific tagging at the reducing end of an oligosaccharide by ${ }^{3} \mathrm{H}$ incorporation, followed by partial deaminative cleavage with dilute $\mathrm{HNO}_{2}$ to yield ${ }^{3} \mathrm{H}-$ labelled intermediate fragments whose sizes reflect positions of the $\mathrm{HNO}_{2}$-sensitive GlcNS- $\alpha(1 \rightarrow 4) \mathrm{HexA}$ linkages (HexA is uronic acid). The fragments are then treated with specific lysosomal enzymes, such as iduronate 2-O-sulphatase (I2Sase [20]), iduronidase (IdoAse [21]) or glucosamine 6- $O$-sulphatase (6Sase [22]) to enable sequence determination from comparison of separation profiles on high-resolution strong-anion-exchange (SAX) HPLC.

\section{EXPERIMENTAL}

Porcine mucosal HS was provided by Organon (Oss, The Nederlands) and the heparin-derived octasaccharide (H8) released by heparinases was generously given by Dr. G. Jayson (Christie Hospital, Manchester, U.K.). Heparinases I, II and III were supplied by Grampian Enzymes (Harray, Orkney, U.K.) and $\Delta$-glycuronidase by Seikagaku (Chuo-Ku, Tokyo, Japan). The recombinant exoenzymes were prepared as previously described [20-22] and stored at $4{ }^{\circ} \mathrm{C}$. They are now available from Oxford Glycosciences (Abingdon, Oxon, U.K.). Bio-Gel P6 and P2 were obtained from Bio-Rad (Hemel Hempstead, Herts., U.K), PD-10 columns from Pharmacia (Little Chalfont, Bucks., U.K.) and Propac PA1 SAX HPLC columns from Dionex (Camberley, Surrey, U.K.). The $\mathrm{NaB}^{3} \mathrm{H}_{4}(33 \mathrm{Ci} / \mathrm{mmol})$ was purchased from Amersham. All other reagents were obtained from BDH-Merck Ltd. (Lutterworth, Leics., U.K.).

\section{Preparation and ${ }^{3} \mathrm{H}$ end labelling of oligosaccharides}

Heparinase III-resistant HS hexasaccharides were prepared by using a two-step purification procedure involving gel filtration (Bio-Gel P6) and SAX-HPLC separation as described previously [9] (see Figure 1 below). Oligosaccharides were detected by measuring UV absorbance at $232 \mathrm{~nm}$, and amounts were determined by weighing. Sample purity was checked by PAGE using Tris/glycine gels (see conditions in Figure 1 below). Oligosaccharides $(20-40 \mathrm{nmol})$ were dissolved in $200 \mu \mathrm{l}$ of $50 \mathrm{mM}$ Tris/ $\mathrm{HCl}, \mathrm{pH} 8.5$, containing $1 \mathrm{mCi}$ of $\mathrm{NaB}^{3} \mathrm{H}_{4}$. After incubation overnight at room temperature, samples were acidified (to $\approx$ $\mathrm{pH} 4$ ) with $4 \mathrm{M}$ acetic acid and then neutralized (to $\approx \mathrm{pH} 7$ ) by addition of $2 \mathrm{M}$ sodium carbonate. Most of the free label was released as ${ }^{3} \mathrm{H}_{2}$ gas during the acidification. Salt and remaining free radioactivity were removed by running the samples on a PD10 column. The radiolabelled oligosaccharides were then purified by SAX-HPLC, using the gradient conditions described in Figure 1 (below). Fractions corresponding to radiolabelled oligosaccharides (determined by liquid-scintillation counting of small aliquots) were pooled and desalted on a PD-10 column. Purity was reassessed by SAX-HPLC.

\section{Specificity of the ${ }^{3} \mathrm{H}$ end labelling}

Radiolabelled HS hexasaccharides were depolymerized by deaminative cleavage with $\mathrm{HNO}_{2}$ using the low-pH method of Shively and Conrad [23]. Deaminated samples were then desalted on a PD-10 column and applied to the Propac PA1 SAX-HPLC column equillibrated in Milli Q water, $\mathrm{pH}$ 3.5. $\left[{ }^{3} \mathrm{H}\right]$ Saccharides were eluted with a two-step linear gradient, $0-0.15 \mathrm{M} \mathrm{NaCl}$ (in Milli Q water, $\mathrm{pH} 3.5$ ) over $51 \mathrm{~min}$ and $0.15-0.5 \mathrm{M} \mathrm{NaCl}$ over $67 \mathrm{~min}$ at a flow rate of $1 \mathrm{ml} / \mathrm{min}$. Peaks were detected by scintillation counting and their elution positions compared with those of radiolabelled disaccharide standards.

The possible presence of ${ }^{3} \mathrm{H}$ label in the non-reducing (unsaturated) uronate was also assessed. Radiolabelled HS hexasaccharides $(\approx 1000$ c.p.m.) were incubated with 1 unit of $\Delta$ glycuronidase in $25 \mu \mathrm{l}$ of $40 \mathrm{mM}$ sodium acetate, $\mathrm{pH} 4.5$, overnight at $37^{\circ} \mathrm{C}$. Digests were subjected to SAX-HPLC and elution times of products were compared with those of untreated oligosaccharides.

\section{Sequence analysis of the ${ }^{3} \mathrm{H}$-end-labelled oligosaccharides}

${ }^{3} \mathrm{H}$-end-labelled oligosaccharides for sequencing were partially depolymerized by $\mathrm{HNO}_{2}\left(\mathrm{pHNO}_{2}\right)$ treatment under mild conditions. Preliminary experiments were carried out to determine incubation times required to yield an appropriate spread of degradative intermediates. For HS saccharides (HS-6A and HS$6 \mathrm{~B}), \approx 10^{5}$ c.p.m. in $80 \mu \mathrm{l}$ of water were incubated with $10 \mu \mathrm{l}$ of $190 \mathrm{mM} \mathrm{HCl}$ and $10 \mu \mathrm{l}$ of $10 \mathrm{mM} \mathrm{NaNO}$. Aliquots $(10-20 \mu \mathrm{l})$ were removed after $0,1,2,3$ and 4 h of incubation and neutralized by the addition of $25 \mu \mathrm{l}$ of $0.2 \mathrm{M}$ sodium acetate, $\mathrm{pH} 4.5$, to stop the degradation. The various aliquots were pooled and desalted on a PD-10 column. For the heparin saccharide H8, which depolymerized more rapidly, a single incubation of 10 min was used. $\mathrm{pHNO}_{2}$-treated oligosaccharides (2000-4000 c.p.m.) were re-dissolved in $25 \mu \mathrm{l}$ of $40 \mathrm{mM}$ sodium acetate, $\mathrm{pH} 4.5$, and incubated for $16-24 \mathrm{~h}$ at $37^{\circ} \mathrm{C}$ with single or combinations of exoenzymes as follows: 1 , I2Sase (0.54 m-units); 2, IdoAse (0.29 munits); 3, I2Sase (0.54 m-units) followed by IdoAse (0.29 m-units); 4, I2Sase (0.54 m-units), then IdoAse (0.29 munits) and finally 6Sase $(0.096 \mu$-units). When combinations were used (i.e. 3 and 4 ), the incubations were performed sequentially in the order stated. Digests were analysed by SAXHPLC using $\mathrm{NaCl}$ linear gradients previously optimized for each starting oligosaccharide (see Figures 3, 4 and 5 below). Fractions $(0.5 \mathrm{ml})$ were analysed for ${ }^{3} \mathrm{H}$ by scintillation counting. Elution patterns of each enzyme digest were compared with the untreated $\mathrm{pHNO}_{2}$ mixture subjected to SAX-HPLC under the same gradient conditions. Mercuric acetate treatment to remove the $\Delta 4,5$ unsaturated uronic acid ( $\triangle \mathrm{HexA}$ residue) at the unsaturated end was performed as previously described [24] and the sample analysed by SAX-HPLC.

\section{Disaccharide analysis of oligosaccharides}

Disaccharide analysis was performed as previously described [9]. Oligosaccharides (unreduced) were degraded extensively by treatment with a mixture of heparinases. Resulting disaccharides were analysed by SAX-HPLC calibrated with authentic standards, using measurement of UV absorbance at $232 \mathrm{~nm}$ for detection.

\section{RESULTS AND DISCUSSION}

\section{Purification of HS-derived hexasaccharides}

The depolymerization of porcine mucosal HS by heparinase III yielded a mixture of sulphated oligosaccharides that were first 
fractionated according to their size using Bio-Gel P6 gel chromatography [9]. Fractions corresponding to the hexasaccharide peak were pooled and the constituent species resolved by SAX-HPLC (Figure 1). Two of the three main peaks in the profile (HS-6A and HS-6B) were taken for sequence analysis. A third oligosaccharide also prepared for sequencing was a single octasaccharide species designated $\mathrm{H} 8$, generated by partial heparinase I digestion of heparin and purified by gel filtration and SAX-HPLC

All three oligosaccharides were first analysed by PAGE to assess their purity. HS-6A and H8 (inset to Figure 1) ran as single bands, whereas HS-6B contained two species: a major component, HS-M6B, and a minor one, HS-m6B, present at a ratio of approx. 3:1 (Figure 1). These samples were radiolabelled by reduction with $\mathrm{NaB}\left[{ }^{3} \mathrm{H}\right]_{4}$ and approximately 10000 d.p.m./nmol of oligosaccharide were incorporated. Free ${ }^{3} \mathrm{H}$ was removed by gel filtration (on a PD-10 column), but a further purification by SAX-HPLC (see the Experimental section) was required to separate the $\left[{ }^{3} \mathrm{H}\right]$ oligosaccharides from radiolabelled non-GAG material (results not shown)

\section{Specificity of ${ }^{3} \mathrm{H}$ end labelling}

The specificity of ${ }^{3} \mathrm{H}$ incorporation at the reducing end is essential for the 'reading' of the saccharide sequence, since it allows the exclusive detection of intermediate-sized fragments that still contain the original reducing end following $\mathrm{pHNO}_{2}$ treatment. Special attention was given to showing that there was no unspecific isotope incorporation as a result of unknown side reactions. First, HS-6B was incubated with $\Delta$-glycuronidase, an exoenzyme that specifically removes unsaturated $\Delta 4,5$-uronic acid. Analysis of the digest by SAX-HPLC showed only one ${ }^{3} \mathrm{H}$ labelled peak corresponding to the resulting pentasaccharide. No free label or radiolabelled monosaccharides were detected (results

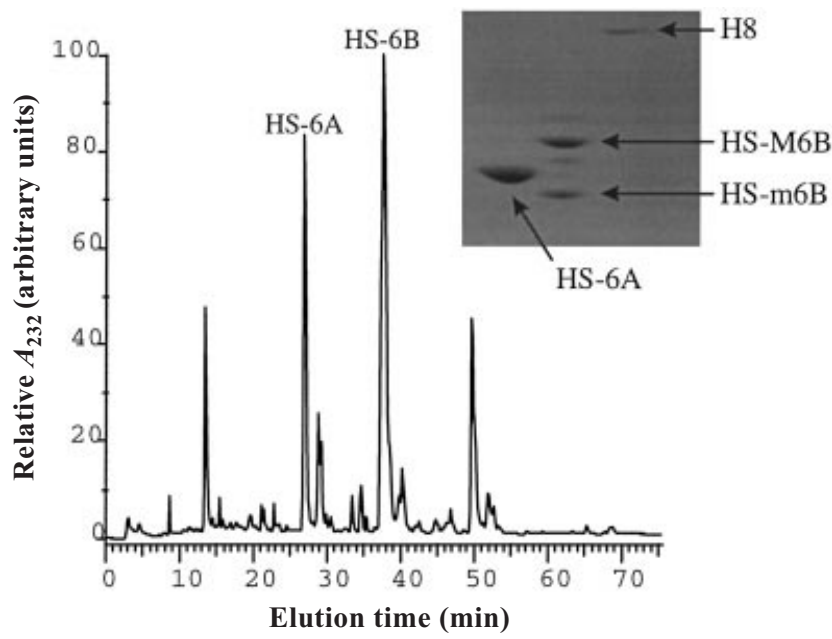

Figure 1 Purification of HS hexasaccharides by SAX-HPLC

Size-defined HS hexasaccharides were applied to two SAX-HPLC Propac PA1 columns connected in series and resolved using a linear gradient of $\mathrm{NaCl}(0.04-2 \mathrm{M} \mathrm{NaCl}$ in Milli $Q$ water, $\mathrm{pH} 3.0$, over $60 \mathrm{~min})$. Peaks were detected by absorbance at $232 \mathrm{~nm}$. Fractions $(1 \mathrm{ml})$ corresponding to the two indicated species (HS-6A and HS-6B) were pooled. Inset: the purity of oligosaccharides was checked by PAGE. HS-6A, HS-6B and H8 were run on a 10-cm-long $30 \%$-(w/v)-acrylamide gel at constant current (25 mA) for 3-4 h. The gel was stained with aq $0.08 \%$ Azure A for 10 min under constant agitation. Excess dye was removed by washing the gel twice in water for $5 \mathrm{~min}$.

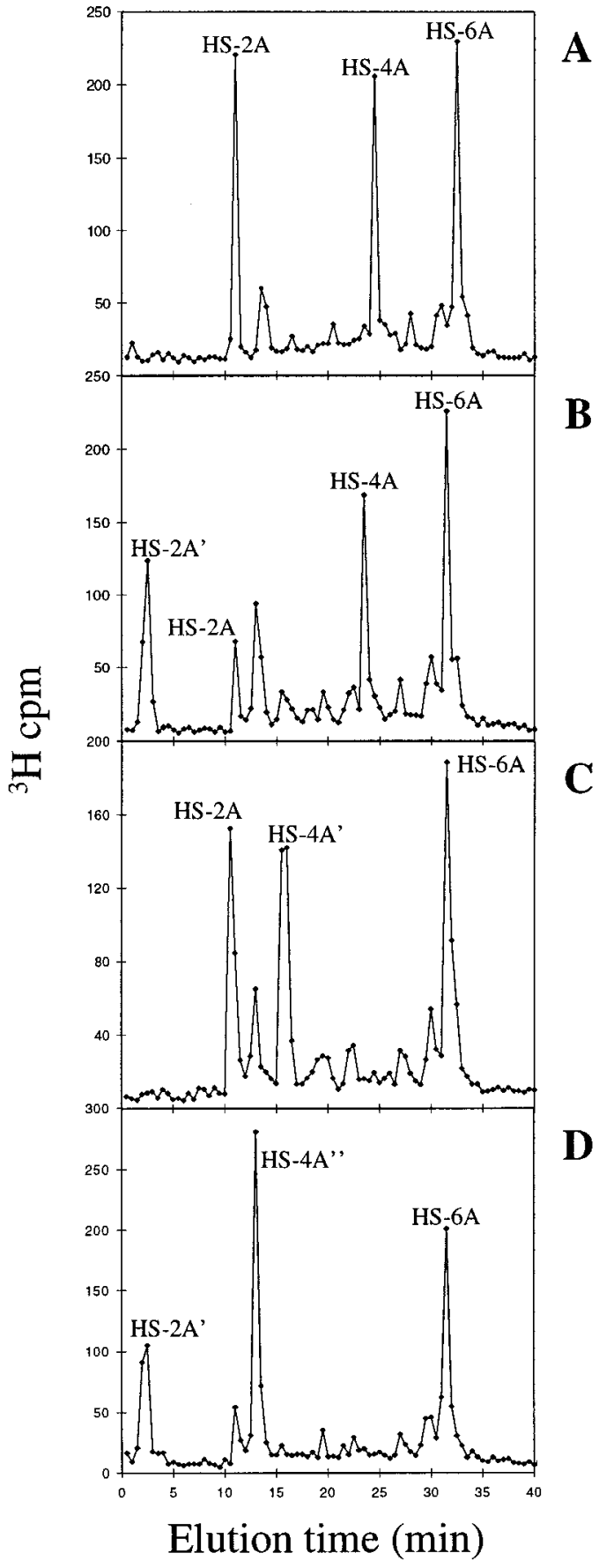

Figure 2 Sequencing of HS-6A

Aliquots (2000 c.p.m.) of $\mathrm{pHNO}_{2}$-treated $\mathrm{HS}-6 \mathrm{~A}$ were incubated with individual or combinations of exoenzymes and analysed by SAX-HPLC. Following a 4 min wash in Milli $Q$ water, $\mathrm{pH} 3.5$ (acidified with $\mathrm{HCl}$ ), samples were eluted using a linear gradient of $\mathrm{NaCl}(0-0.7 \mathrm{M} \mathrm{NaCl}), \mathrm{pH}$ 3.5, over $38 \mathrm{~min}$ at $1 \mathrm{ml} / \mathrm{min}$. pHNO ${ }_{2}$-treated HS-6A (A) was digested with IdoAse (B), I2Sase (C), I2Sase + IdoAse (D)

not shown). Secondly, complete $\mathrm{HNO}_{2}$ depolymerization of both HS hexasaccharides generated a single radiolabelled disaccharide for HS-6A and, two for HS-6B with a 3:1 ratio (results not shown), thus showing that the two distinct species seen in HS-6B (Figure 1) differ at their reducing end. 


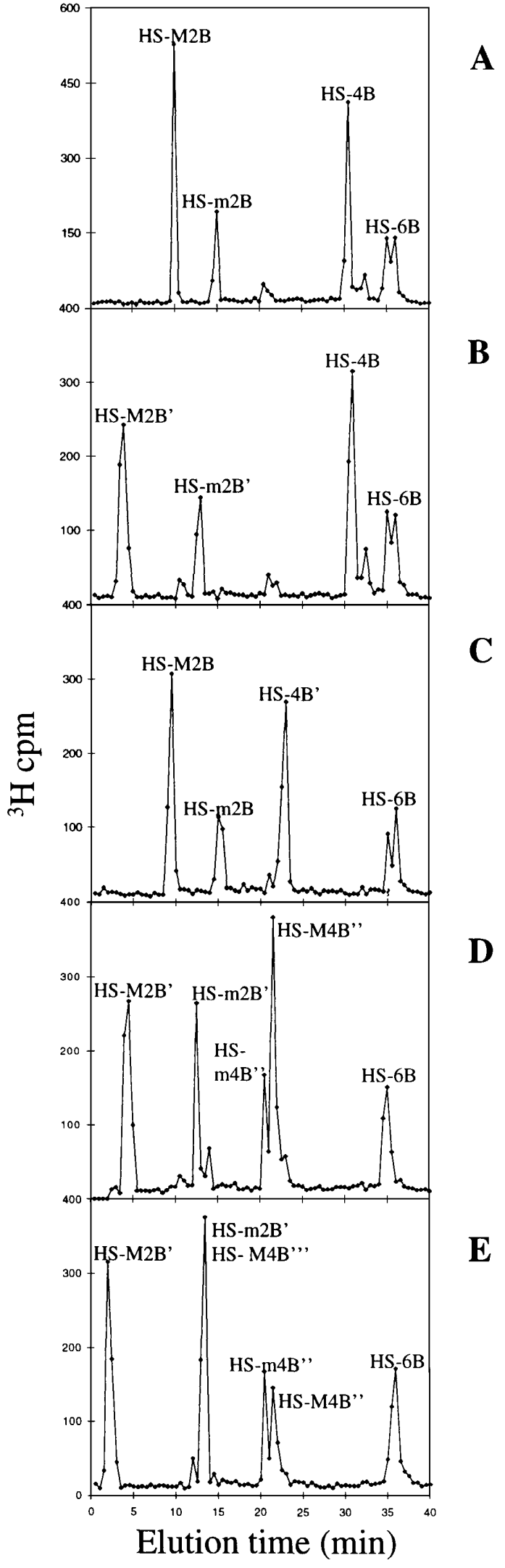

Figure 3 Sequencing of HS-6B

Aliquots (2000 c.p.m.) of $\mathrm{pHNO}_{2}$-treated $\mathrm{HS}-6 \mathrm{~B}$ were incubated with exoenzymes and analysed by SAX-HPLC. After a 4 min wash in Milli $Q$ water, pH 3.5, samples were eluted using a linear gradient of $\mathrm{NaCl}(0-0.8 \mathrm{M} \mathrm{NaCl})$, pH 3.5, over $38 \mathrm{~min}$ at $1 \mathrm{ml} / \mathrm{min}^{\mathrm{p}} \mathrm{pHNO}{ }_{2}$-treated $\mathrm{HS}-6 \mathrm{~B}(\mathbf{A})$ was digested with IdoAse (B), I2Sase (C), I2Sase + IdoAse (D), I2Sase + IdoAse +6Sase (E). In (E) a small fraction of the HS-M4B" peak (D) is not converted into HS-4MB" by 6Sase.
Table 1 Disaccharide (DS) analysis of heparin and HS oligosaccharides

Key to oligosaccharides (OS): 1, $\triangle$ HexUA-GlcNAc; 2, $\Delta$ HexUA-GIcNAc(6S); 3, $\Delta$ HexUA$\mathrm{GlcNSO}_{3} ; 4, \Delta \mathrm{HexUA-GlcNS}(6 \mathrm{~S}) ; 5, \Delta \mathrm{HexUA(2S)-GlcNSO}{ }_{3} ; 6, \Delta \mathrm{HexUA(2S)-GlcNSO3(6S)}$; 7, $\triangle$ HexUA (2S)-GIcNAc. Abbreviation: nd, not detected.

\begin{tabular}{llllllllll}
\hline \multicolumn{7}{c}{ Disaccharide (\% of total) } & & & \\
DS & OS ... & 1 & 2 & 3 & 4 & 5 & 6 & 7 \\
\hline HS-6A & & 26.1 & 3.4 & 35.7 & 1.2 & 32.4 & 1.2 & nd \\
NS-6B & 26.1 & 6.5 & 31.4 & 1.8 & 8 & 25.5 & nd \\
H8 & nd & nd & nd & nd & nd & 100 & nd \\
& & & & & & & & \\
\hline
\end{tabular}

\section{Sequencing of ${ }^{3} \mathrm{H}$-end-labelled oligosaccharides}

Analysis of HS-6A hexasaccharide

Partial depolymerization of HS-6A with $\mathrm{HNO}_{2}$ resulted in the generation of three main peaks corresponding to the reducingend-derived disaccharide (HS-2A), tetrasaccharide (HS-4A) and the remaining intact hexasaccharide (Figure 2A), suggesting, therefore, that both the internal amino sugars were $\mathrm{N}$-sulphated. Thus the general structures of the $\mathrm{pHNO}_{2}$ fragments are $(\mathrm{SP}=$ sugar position):

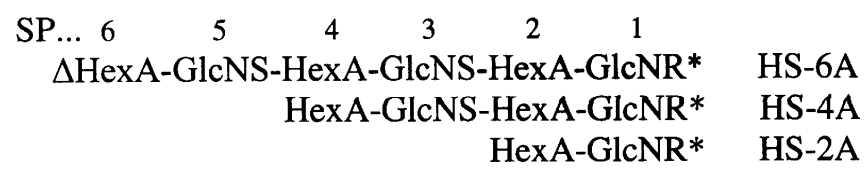

in which $\mathrm{R}$ at residue 1 is $\mathrm{Ac}$ or $\mathrm{SO}_{3}^{-}$, * indicates ${ }^{3} \mathrm{H}$-labelled glucosaminitol, the HexA represents GlcA or IdoA, the uronates may be $\mathrm{O}$-sulphated at $\mathrm{C}-2$ and the amino sugars at $\mathrm{C}-6$. The $\mathrm{pHNO}_{2}$ scission not only reveals the position of GlcNS but also 'opens up' the internal sequence to the action of lysosomal exoenzymes. The $\mathrm{pHNO}_{2}$ fragments were then incubated with exoenzymes, singly or in combination, and digestion products analysed by SAX-HPLC (see Figure 2). The incubation with IdoAse (Figure 2B) specifically degraded HS-2A to HS-2A' (identified as $\left[{ }^{3} \mathrm{H}\right] \mathrm{GlcNAc-ol}$ ), defining the uronic acid at position 2 in the above sequence as IdoA. Digestion with I2Sase (Figure 2C) resulted in a specific shift only of the HS-4A peak to HS-4A', indicating the presence of a $2-O$-sulphate group on the uronic acid at position 4 . The combination of both I2Sase and Idoase (Figure 2D) converted HS-4A into HS-4A", a trisaccharide derived from HS-4A after removal of the IdoA,2S at position 4. Finally, incubation of the sample with three exoenzymes successively (I2Sase, IdoAse and 6Sase) caused no further movement of the peaks when compared with the I2Sase + IdoAse profile (Figure 2D), suggesting the absence of 6-O-sulphate groups in HS-6A (results not shown). The HS-6A sequence that can be read from the profiles in Figure 2 is:

\section{$\Delta$ HexA-GlcNS-IdoA,2S-GlcNS-IdoA-GlcNAc}

\section{Analysis of HS-6B hexasaccharide}

For HS-6B, two different structures (in a ratio of $3: 1$ ) were sequenced at the same time. In the $\mathrm{pHNO}_{2}$ digest (Figure $3 \mathrm{~A}$ ), one tetrasaccharide (HS-4B) and two disaccharides were observed together with the original HS-6B, hence showing the internal glucosamines to be N-sulphated. The disaccharide derived from 


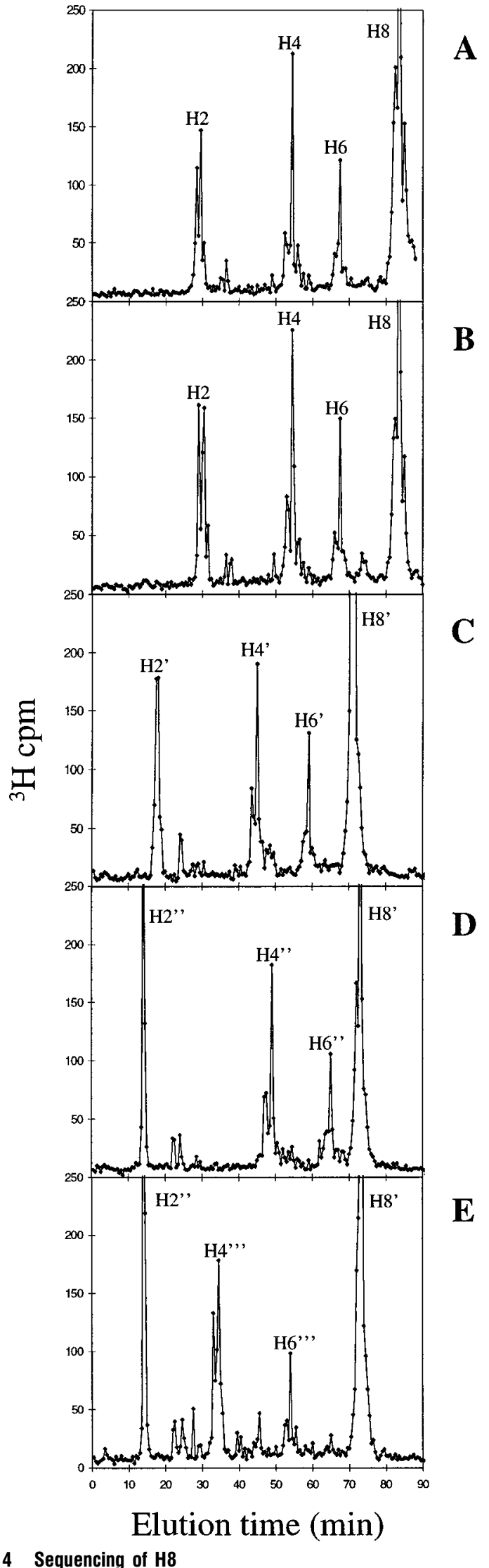

Figure 4 Sequencing of $\mathrm{H} 8$

Aliquots (3000 c.p.m.) of $\mathrm{pHNO}_{2}$-treated $\mathrm{H} 8$ were incubated with exoenzymes and analysed by SAX-HPLC. Following a 4 min wash in Milli $Q$ water, $\mathrm{pH}$ 3.5, samples were eluted using a linear gradient of $\mathrm{NaCl}(0-1.7 \mathrm{M} \mathrm{NaCl})$, pH 3.5, over $80 \mathrm{~min}$ at $1 \mathrm{ml} / \mathrm{min}$. $\mathrm{PHNO}_{2}$-treated $\mathrm{H} 8$ (A) was digested with IdoAse (B), I2Sase (C), I2Sase + IdoAse (D), I2Sase + IdoAse +6Sase (E). the major species (HS-M2B) was eluted before the one derived from the minor component (HS-m2B). Digestion of the $\mathrm{pHNO}_{2}$ fragments with IdoAse affected only the disaccharides (Figure 3B), HS-M2B and HS-m2B being converted into HS-M2B' (corresponding to GlcNAc-ol) and HS-m2B' respectively, which showed that residues at position 2 (adopting the numbering system used for HS-6A) in both species are IdoA (Figure 3). Treatment with I2Sase converted HS-4B into HS-4B' (Figure $3 \mathrm{C}$ ), showing the presence of a 2-O-sulphate group on the uronate at position 4. Combined use of I2Sase and IdoAse (Figure 3D) generated two distinct trisaccharides from HS-4B with one (HS-M4B") more abundant than the other (HS-m4B"). This indicated that residue 4 is IdoA,2S for both species. Finally, consecutive digestions with I2Sase, IdoAse and 6Sase (Figure $3 \mathrm{E})$ generated a new derivative, HS-M4B"', a product derived from HS-M4B", hence revealing a $6-O$-sulphate group on the number 3 glucosamine of the major species HS-M6B. This fragment (HS-M4B"') was eluted at the same position as the monosaccharide HS-m2 $\mathbf{B}^{\prime}$ and so masked this latter species. Therefore the digestion was repeated on HPLC-purified HS-4B and HS-m2B, which confirmed the co-elution of the products HS-M4B"' and HS-m2B' (results not shown). It was also observed that the monosaccharide HS-m2 $B^{\prime}$ generated by IdoAse treatment was not affected by the 6Sase; however, its elution position is indicative of a monosulphated sugar, and the disaccharide analysis performed on HS-6B (Table 1) suggested that the residue at position 1 of the minor species is GlcNAc, $6 \mathrm{~S}$. We therefore assume that the 6Sase will not act on reduced, open-ring 6-Osulphated substrates. The sequences of the two oligosaccharides found in HS-6B and their respective $\mathrm{pHNO}_{2}$ fragments can therefore be read from the profiles shown in Figure 3, and they are:

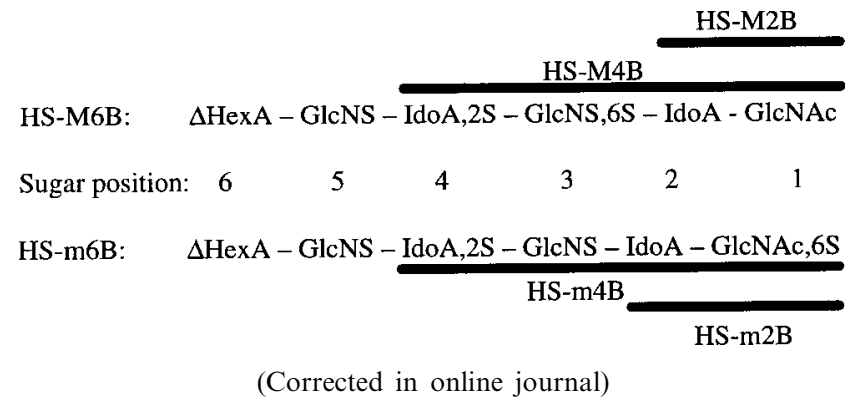

Analysis of heparin octasaccharide $(\mathrm{H} 8)$

The $\mathrm{pHNO}_{2}$ elution profile for the heparin octasaccharide $\mathrm{H} 8$ showed four main peaks corresponding to the intact $\mathrm{H} 8$ and three intermediates $(\mathrm{H} 2, \mathrm{H} 4$ and $\mathrm{H} 6$ corresponding to di-, tetraand hexa-saccharides respectively; Figure 4A). Exoenzyme digestions of $\mathrm{pHNO}_{2}$-treated $\mathrm{H} 8$ showed that IdoAse alone was ineffective on any of the peaks generated by $\mathrm{pHNO}_{2}$ (Figure $4 \mathrm{~B}$ ), whereas I2Sase caused shifts in elution position of all four species, i.e. $\mathrm{H} 2, \mathrm{H} 4, \mathrm{H} 6$ and $\mathrm{H} 8$ to $\mathrm{H} 2^{\prime}, \mathrm{H}^{\prime}, \mathrm{H}^{\prime}$ and $\mathrm{H}^{\prime}$ respectively (Figure 4C), revealing the presence of $2-O$-sulphates on all uronic acids. Interestingly, it was noticed in this digestion that I2Sase also accepted unsaturated uronic acids as substrates, since $\mathrm{H} 8$ was also affected by the enzyme. Following I2Sase treatment, IdoAse was able to act on $\mathrm{H}^{\prime}, \mathrm{H}^{\prime}$ ' and $\mathrm{H}^{\prime}$ ' converting them into species designated $\mathrm{H} 2^{\prime \prime}, \mathrm{H} 4^{\prime \prime}$ and $\mathrm{H}^{\prime \prime}$ respectively (Figure 4D). This result established the nature of all the internal uronic acids as IdoA residues. Surprisingly, H4" and H6" were eluted at a higher salt concentration than their substrates, $\mathrm{H}^{\prime}$ and $\mathrm{H}^{\prime}$ respectively, probably as a result of their higher charge 


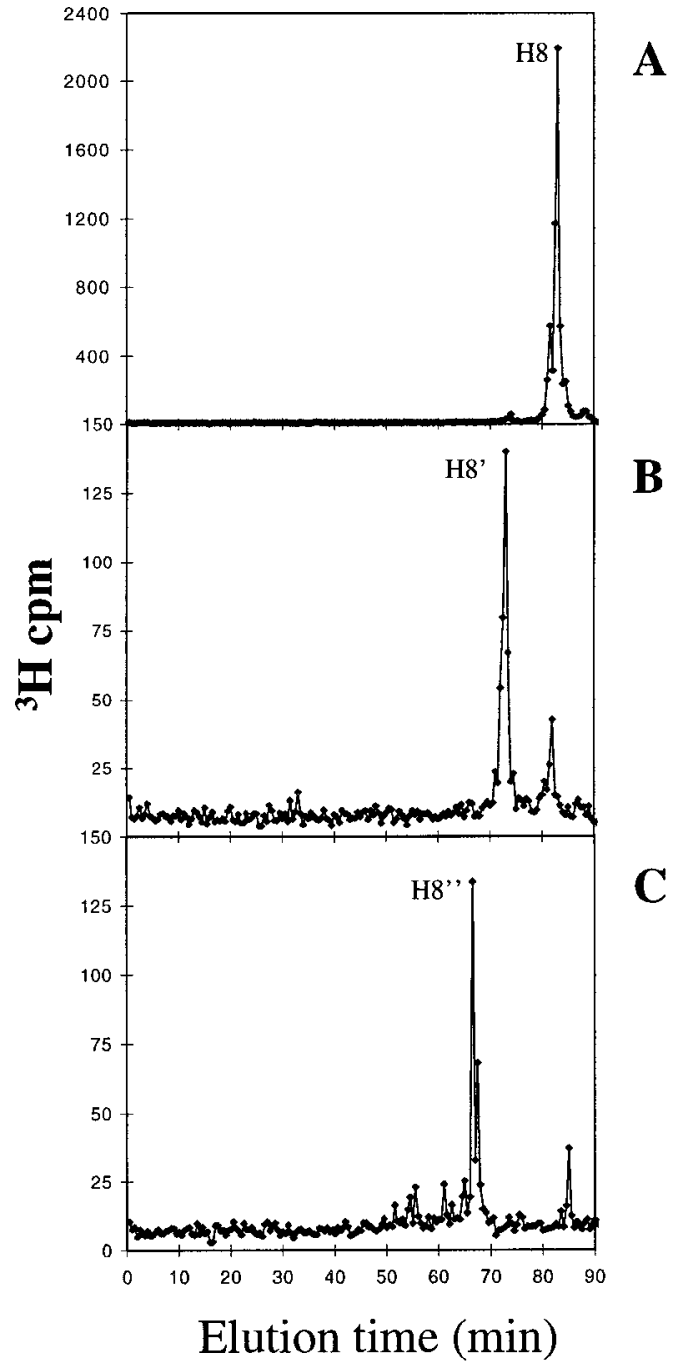

Figure 5 Identification of the unsaturated disaccharide of $\mathbf{H 8}$

Radiolabelled $\mathrm{H} 8$ was treated with mercuric acetate, desalted and digested with 6Sase. Resulting products were analysed by SAX-HPLC. (A) H8; (B) H8 treated with mercuric acetate; (C) H8 treated with mercuric acetate and 6Sase.

density. The $\mathrm{H}^{\prime}$ fragment was unaffected by IdoAse, which is unable to remove an unsaturated uronate residue. Finally, the digestion of the $\mathrm{pHNO}_{2}$ fragments by all three exoenzymes successively (i.e. I2Sase, IdoAse, 6Sase) generated two new components, $\mathrm{H}^{\prime \prime \prime}$ and $\mathrm{H}^{\prime \prime \prime}$, showing the presence of 6-Osulphates on both internal glucosamines at positions 3 and 5 (Figure 4E). The H2" monosaccharide was not acted on by the 6Sase (Figures 4D and 4E); however, the elution position of this monosaccharide and the disaccharide analysis of $\mathrm{H} 8$ (Table 1) show that the residue 1 is GlcNS,6S.

The 6-O-sulphation of the glucosamine residue next to the $\Delta \mathrm{HexA}, 2 \mathrm{~S}$ at the non-reducing end of $\mathrm{H} 8$ was then investigated by treatment of the intact $\mathrm{H} 8$ with mercuric acetate to remove the $\triangle \mathrm{HexA}, 2 \mathrm{~S}[24]$ and digestion by the 6 Sase. Digests were analysed by SAX-HPLC (Figure 5). Mercuric acetate converted H8 into the derived heptasaccharide $\mathrm{H} 8^{\prime}$ (Figure 5B). The latter was then digested by the 6Sase to give $\mathrm{H} 8$ " (Figure 5C), showing the presence of a $6-O$-sulphate group on the non-reducing-end disaccharide. The sequence of $\mathrm{H} 8$ was thus established to be a succession of four trisulphated disaccharides:

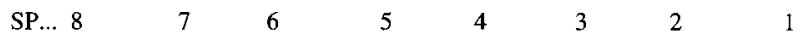

$\triangle$ HexA,2S-GlcNS,6S-IdoA,2S-GlcNS,6S-IdoA,2S-GlcNS,6S-IdoA,2S-GlcNS,6S

(Corrected in online journal)

Disaccharide composition of the oligosaccharides

The composition of the oligosaccharide preparations analysed in this study (Table 1) matched the data provided by the sequence analysis and gave the expected proportions of the constituent disaccharide units. For HS-6A, three different disaccharides in equal proportions were identified. All were devoid of 6-Osulphate groups, and only one contained a 2-O-sulphated uronate. Disaccharide analysis of the heterogeneous sample HS6B showed five disaccharides to be present. The ratio of $3: 1$ between the two hexasaccharides present enabled attribution of disaccharides to one species or the other, with one disaccharide ( $\triangle$ HexA-GlcNS) being common to both species. Once again, the nature of the disaccharides identified confirmed the sequences proposed: only two disaccharides were found to contain a 6-Osulphate group, the major one being $\triangle \mathrm{HexA}, 2 \mathrm{~S}-\mathrm{GlcNS}, 6 \mathrm{~S}$ (from HS-M6B), while the less abundant one is $\Delta$ HexA-GlcNAc, $6 \mathrm{~S}$ (from HS-m6B). Finally, only the trisulphated disaccharide ( $\Delta \mathrm{HexA}, 2 \mathrm{~S}-\mathrm{GlcNS}, 6 \mathrm{~S}$ ) was found in $\mathrm{H} 8$, supporting the structure deduced for this oligosaccharide.

\section{CONCLUSION}

The method described here enables fast and reliable sequencing of small amounts (approx. $20 \mathrm{nmol}$ ) of HS and heparin oligosaccharides. The high-resolution SAX-HPLC separation of fragments of intermediate size generated by $\mathrm{pHNO}_{2}$ enabled positioning of the GlcNS residues and a clear revelation of shifts in elution position of the fragments after exoenzyme treatment. Hence, there is no need to separate individual saccharides after $\mathrm{pHNO}_{2}$ scission, as these can be analysed simultaneously, so greatly accelerating the speed and efficiency of sequencing. We have also demonstrated that the technique can accommodate a certain level of contamination; i.e. in the case of HS-6B, the sequencing of two species was achieved. Three exoenzymes (I2Sase, IdoAse and 6Sase) were needed for sequencing our oligosaccharides. However, the use of other exoenzymes which are currently available or still under development, should enable characterization of some rarer saccharides found in HS/heparin [20]. Furthermore, this technique could easily be adapted for the sequencing of other GAGs such as chondroitin sulphate or dermatan sulphate using alternative scission methods and specific exoenzymes. We believe that this technique will have considerable applications in the growing field of GAG-binding proteins and will aid our understanding of the complex structure-function relationships of $\mathrm{HS}$.

We thank Dr. G. Jayson for the heparin octasaccharide H8, Dr. G. Van Deedem (Organon Co.) for porcine mucosal HS, Kaye Beckman and Chris Boulter for help with preparation of exoenzymes. This work was supported by grants from the Cancer Research Campaign (U.K.), the National Health and Medical Research Council of Australia, the Swedish Medical Research Council (2309, 1296-03), the Swedish Cancer Society (Grant 3919-B97) and the Association SANOFI Thrombose pour la recherche (France).

\section{REFERENCES}

1 Gallagher, J. T., Lyon, M. and Steward, W. .P. (1986) Biochem. J. 236, 313-325

2 Gallagher, J. T., Turnbull, J. E. and Lyon, M. (1992) Int. J. Biochem. 24, 553-560

3 Salmivirta, M., Lidholt, K. and Lindahl, U. (1996) FASEB. J. 10, 1270-1279 
4 Lindahl, U., Kusche-Gullberg, M. and Kjellen, L. (1998) J. Biol. Chem. 273 24979-24982

5 Kjellen, L. and Lindahl, U. (1991) Annu. Rev. Biochem. 60, 443-475

6 Bourin, M. C. and Lindahl, U. (1993) Biochem. J. 289, 313-330

7 Turnbull, J. E., Fernig, D. G., Ke, Y., Wilkinson, M. C. and Gallagher, J. T. (1992) J. Biol. Chem. 267, 10337-10341

8 Maccarana, M., Casu, B. and Lindahl, U. (1993) J. Biol. Chem. 268, 23898-23905

9 Pye, D. A., Vives, R. R., Turnbull, J. E., Hyde, P. and Gallagher, J. T. (1998) J. Biol. Chem. 273, 22936-22942

10 Guimond, S., Maccarana, M., Olwin, B. B., Lindahl, U. and Rapraeger, A. C. (1993) J. Biol. Chem. 268, 23906-23914

11 Ishihara, M. (1994) Glycobiology 4, 817-824

12 Lyon, M., Deakin, J. A., Mizuno, K. and Gallagher, J. T. (1994) J. Biol. Chem. 269, $11216-11223$

13 Tessler, S., Rockwell, P., Hicklin, D., Cohen, T., Levi, B. Z., Witte, L., Lemischka, I. R. and Neufeld, G. (1994) J. Biol. Chem. 269, 12456-12461

14 Turnbull, J. E. and Gallagher, J. T. (1991) Biochem. J. 273, 553-559

15 Lyon, M., Deakin, J. A. and Gallagher, J. T. (1994) J. Biol. Chem. 269, 11208-11215

Received 8 February 1999/23 February 1999; accepted 2 March 1999
16 Maccarana, M., Sakura, Y., Tawada, A., Yoshida, K. and Lindahl, U. (1996) J. Biol. Chem. 271, 17804-17810

17 Chai, W., Hounsell, E. F., Bauer, C. J. and Lawson, A. M. (1995) Carbohydr. Res. 269, 139-156

18 Rhomberg, A. J., Ernst, S., Sasisekharan, R. and Biemann, K. (1998) Proc. Natl. Acad. Sci. U.S.A. 95, 4176-4181

19 Turnbull, J. E., Hopwood, J. J. and Gallagher, J. T. (1997) in A Laboratory Guide to Glycoconjugate Analysis (Jackson, P. and Gallagher, J. T., eds.), pp. 261-277, Birkhäuser, Basel

20 Bielicki, J., Hopwood J..J., Wilson P. J. and Anson, D. S. (1992) Biochem. J. 289, $241-246$

21 Unger, E. G., Durrant J., Anson D. S. and Hopwood, J. J. (1994) Biochem. J. 304, 43-49

22 Litjens, T., Bielicki J., Anson D. S., Friderici K., Jones M. Z. and Hopwood, J. J. (1997) Biochem. J. 327, 89-94

23 Shively, J. E. and Conrad, H. E. (1976) Biochemistry 15, 3932-3942

24 Ludwigs, U., Elgavish, A., Esko, J. D., Meezan, E. and Roden, L. (1987) Biochem. J. 245, 795-804 